\title{
Role of Tumor Necrosis Factor-Producing Mesenchymal Stem Cells on Apoptosis of Chronic B-lymphocytic Tumor Cells Resistant to Fludarabine-based Chemotherapy
}

\author{
Armita Valizadeh ${ }^{1}$, Ahmad Ahmadzadeh $^{2}$, Ghasem Saki ${ }^{1 *}$, Ali Khodadadi ${ }^{3}$, Ali \\ Teimoori $^{3}$
}

\begin{abstract}
Background: B-cell chronic lymphocytic leukemia B (B-CLL), the most common type of leukemia, may be caused by apoptosis deficiency in the body. Adipose tissue-derived mesenchymal stem cells (AD-MSCs) as providers of pro-apoptotic molecules such as tumor necrosis factor-related apoptosis-inducing ligand (TRAIL), can be considered as an effective anti-cancer therapy candidate. Therefore, in this study we assessed the role of tumor necrosis factor-producing mesenchymal stem cells oin apoptosis of B-CLL cells resistant to fludarabinebased chemotherapy. Materials and Methods: In this study, after isolation and culture of AD-MSCs, a lentiviral LeGO-iG2-TRAIL-GFP vector containing a gene producing the ligand pro-apoptotic with plasmid PsPAX2 and PMDG2 virus were transfected into cell-lines to generate T293HEK. Then, T293HEK cell supernatant containing the virus produced after 48 and 72 hours was collected, and these viruses were transduced to reprogram AD-MSCs. Apoptosis rates were separately studied in four groups: group 1, AD-MSCs-TRAIL; group 2, AD-MSCs-GFP; group 3, AD-MSCs; and group 4, CLL. Results: Observed apoptosis rates were: group 1, $42 \pm 1.04 \%$; group 2, $21 \pm 0.57 \%$; group $3,19 \pm 2.6 \%$; and group $4, \% 0.01 \pm 0.01$. The highest rate of apoptosis thus occurred ingroup 1 (transduced TRAIL encoding vector). In this group, the average medium-soluble TRAIL was $72.7 \mathrm{pg} / \mathrm{m}$ and flow cytometry analysis showed a pro-apoptosis rate of $63 \pm 1.6 \%$, which was again higher than in other groups. Conclusions: In this study we have shown that tumor necrosis factor (TNF) secreted by AD-MSCs may play an effective role in inducing B-CLL cell apoptosis.
\end{abstract}

Keywords: B-cell chronic lymphocytic leukemia B cells - mesenchymal stem cells - TNF - chemotherapy resistance

Asian Pac J Cancer Prev, 16 (18), 8533-8539

\section{Introduction}

B-cell chronic lymphocytic leukemia (B-CLL) is one of the most common leukemia in Western countries and affects more men than women (Hallek et al., 2008). In this type of leukemia, the body's cells show defective apoptotic processes and have a long-life, and eventually colonies of B-cells are formed in peripheral blood, lymphoid organs, and bone marrow (Kay et al., 2007; Hallek., 2015). Celltherapy is one of the therapies that have attracted the attention of many researchers in leukemia treatments. One of the cells used in the treatment of cancer is mesenchymal stem cells (MSCs) (Loebinger et al., 2010). These cells are capable to act as a carrier vehicle to deliver antitumor molecules to tumor site.

Adipose tissue-derived mesenchymal stem cells (ADMSCs) are considered as a promising cellular source for many treatments protocols (Sen et al., 2001; Pachon et al., 2011). Many number of AD-MSCs is simply derived from adipose tissue compared to bone marrow mesenchymal stem cells (BM-MSCs) using a non-invasive method. ADMSCs can code tumor necrosis factor-related apoptosisinducing ligand (TRAIL) against cancer cells (Grisendi et al., 2010). TRAIL is considered as a promising candidate for cancer therapy (Ashkenazi et al., 2008). TRAIL is a type 2 transmembrane death ligand that induces apoptosis in various human cancers, but not in normal cells. In fact, this ligand is a part of the immune system that prevents the formation and spread of tumors (Wiley et al., 1995; Mahalingam et al., 2009). This ligand triggers apoptosis on binding to the death receptors (DR) (Walczak et al., 2008). If TRAIL enters the body intravenously, it quickly disappears due to its short pharmacokinetic half-life (de et al., 2003; Ashkenazi et al., 2008).

According to the clinical application of stem cells and vectors in the treatment of cancer, and the lack of response to cancer chemotherapy, in the present study vectors are used as a carrier to transfer, increase and the durability of

${ }^{1}$ Physiology Research Center, School of Medicine, ${ }^{2}$ Thalassemia and Hemoglobinopathies Research Center, Shafa Hospital, ${ }^{3}$ Cancer, Petroleum and Environmental Pollutants Research Center, Ahvaz Jundishapur University of Medical Sciences, Ahvaz, IR Iran *For correspondence: ghasemsaki@yahoo.com 
the expression of gene responsible for TRIAL. Therefore, in this study we assessed the role of tumor necrosis factorproducing mesenchymal stem cells on apoptosis of B-CLL cells resistant to fludarabine-based chemotherapy.

\section{Materials and Methods}

\section{Isolation and cultivation of stem cells}

In this experimental study, samples of adipose tissue from the abdomen hypodermis were prepared. The samples were collected from patients under age 30 . Then, sterile adipose tissue was put into phosphate buffered saline (PBS) and penicillin- streptomycin antibiotics (GIBCO). After careful washing and precise removal of the blood vessels and debris, adipose tissue was transferred into other sterile plates. Adipose tissue was completely crushed and three times its size $0.1 \%$ collagenase was added to the solution, and then was peptidized. The solution has been transferred to Falcon tubes and was incubated for 30 minutes in the shaking incubator at $37^{\circ} \mathrm{C}$. The solution was centrifuged for $5 \mathrm{~min}$ at $3000 \mathrm{rpm}$. Cell decomposition was cultured with $2 \mathrm{ml}$ DMEM medium and was peptidized with serum FBS $10 \%$. The cells were poured into the flask, the culture medium was added to the flask, and finally the solution was incubated. Isolated MSC was inactivated in a culture DMEM medium containing high glucose, pyruvate and L-glutamine, serum FBS 10\%, then was cultured in $3.7 \mathrm{~g} / \mathrm{L}$ sodium bicarbonate, non-essential amino acids $1 \%$, penicillin $(100 \mu \mathrm{g} / \mathrm{ml})$ and streptomycin $(100 \mu \mathrm{g} / \mathrm{ml})$. Then, cells were incubated for $72 \mathrm{~h}$ at $37^{\circ}$ $\mathrm{C}$ and $95 \%$ humidity and 5\% CO2. Now, MSCs adhered completely to the bottom of the flask; thus, non-adherent cells were separated from the rest of the cells. However, after four days of cell culture, medium was replaced with fresh medium and non-adherent cells were removed, and cell flask was transferred back into the incubator. At this stage of growth, the cells were examined under a light invert microscope.

Passage of adipose tissue-derived mesenchymal stem cells (AD-MSCs)

for cell passage, culture medium was removed and the cells culture plate was washed with PBS once. Furthermore, Trypsin-EDTA $0.25 \%$ enough to cover the surface of cells was added, then, was kept at room temperature until the cells separate from the bottom of the container and the cells were mechanically pipette. To disable Trypsin-EDTA, 15\%FBS with DMEM was used. After pipetting the cell, the contents of cells culture plate were centrifuged at $1500 \mathrm{rpm}$ for $5 \mathrm{~min}$ at $4^{\circ} \mathrm{C}$. Then, cells were transferred to new culture flasks. After three passages, confirmation of nature of cultured MSCs was performed.

\section{Confirmation of nature of cultured MSC}

To confirm the stromal nature of cultured cells, fluorochrome-conjugated monoclonal cocktails antibodies with antigens CD90 and CD105 as MSCs markers and antigens CD45 and CD34 as hematopoietic cell markers with proper control isotypes and flow cytometry (Becton Dickinson, San Jose, CA, USA) were used (Sadighi et al.,
2014). In short, using Trypsin-EDTA $25.0 \%$, cells were isolated from the flasks floor and for the confirmation of each antibody 105 cells were cast within a clean Eppendorf tube, as well as for each antibody an isotype control tube was considered. Then, cells were washed once with cold PBS containing $2 \%$ FBS. Finally, the stained cells were examined by flow cytometry and Win MDI 2.8 software (BD, Franklin Lakes, NJ, USA). Differentiation of MSC into bone cells and adipose tissue was done similar to that provided by Sidighi et al (Sidighi et al., 2014).

\section{Design and construction of TRAIL gene}

In order to construct a recombinant vector encoding a gene expression vector LeGO-IG2 to TRAIL (GFPTRAILLeGO-IG2), TRAIL protein-coding sequence was extracted from the GenBank database based on the reference accession number NM_003810.3 (Abdul et al., 2014). In addition, TRAIL, was digested by BamHI and ECORI restriction enzymes, to clone in 30432pGH plasmid. Then, TRAIL was sequenced (nedagene.com). TRAIL synthesized in pGH vector (TRAIL-30432pGH) was purchased as TNF lyophilized powder $(4 \mu \mathrm{g}$ powder dissolved with $50 \mathrm{ml} \mathrm{TE}$ buffer, and then stored at $-20^{\circ} \mathrm{C}$ ).

\section{Purifying plasmid from positive bacterial colonies}

At this stage, mini preparation plasmid purification was performed. Plasmid purification was performed using a plasmid extraction miniprep kit (QIA gene). Purity and concentration of extracted plasmid were measured both by electrophotometer and agarose gel electrophoresis.

\section{Investigation of DNA on agarose gels}

The extracted DNA fragments DNA was analyzed on $1 \%$ agarose gel.

\section{Double digested plasmid TRAIL-30432pGH}

gene plasmid carries was greatly amplified, to ensure the existence of a DNA fragment in the transformed bacterial following plasmids extraction, enzymatic digestion was performed on them. In the enzymatic digestion, to verify the performance of each enzyme and getting information about the star activity, double digestion was done. Enzymatic digestion was performed in a nuclease-free Eppendorf tube. Moreover, enzymatic digestion products were run under electrophoresis with the 100 bps molecular weight marker on $1 \%$ agarose gel. To eliminate non-specific bands in the gel electrophoresis of the vector, in this study, a gel extraction commercial kit (QIAgene) were used.

\section{Statistical analysis}

Quantitative data were analyzed using Kruskal-Wallis and one-way analysis methods. Post hoc Tukey's test was used to perform between and within group analysis. Data were presented as mean \pm SEM. Besides, data were analyzed using EXELL 2010 and SPSS 19 softwares. Significance level of $\mathrm{P}$ was considered as $\leq 0.05$.

\section{Results}

Isolation and culture of human AD-MSCs 
TNF-Producing Mesenchymal Stem Cells and Apoptosis of Chronic B-Lymphocytic Tumor Cells Resistant to Chemotherapy

AD-MSCs after a day in DMEM with $10 \%$ FBS serum showed fusiform appearance, although in a heterogeneous population of cells, as well as round cells, suspended cells and RBC were observed (Figure 1A). Spindle-shaped cells grow more than other cells and it was the most frequent cell formed after the first passage (Figure B1). ADMSCs in the next passages showed a more homogeneous population of spindle cells were occupied the bottom of the flask (Figure C1).

Confirmation of the nature of MSCs that were cultured using cell surface markers

Around $91.54 \%$ and $96.36 \%$ of these cells expressed the CD105 and CD90 markers on their surface, respectively (Figure 2). These cells were negative for markers of hematopoietic cell as well, and about $1.10 \%$ of these cells expressed CD34 marker on their surface (Figure 2). Besides, about $4 \%$ of the cells express the CD45 markers on their surface (Figure 2).

Confirmation of the nature of the cultured MSCs with osteogenic and adipogenic differentiation

Over the osteogenic medium cell death rate was very high. In addition, during the days of cell culture, cell
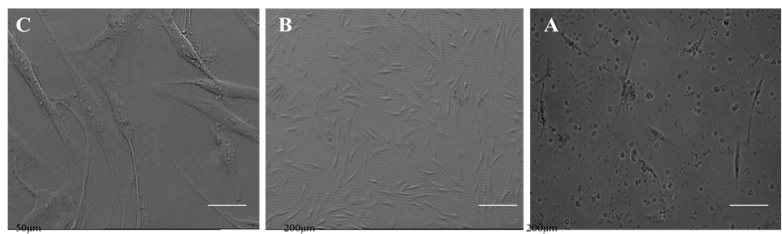

Figure 1. A, Initial Population of Cells Isolated from Adipose Tissue after the First Passage; B, in the Fourth Passage the Number of Spindle Cell Population Began to Increase and their Growth Rate Increases; $C$, in the Fourth Passage Uniformity of Spindle-shaped Cells with Distinct Nuclei was Observed $(\times 40)$
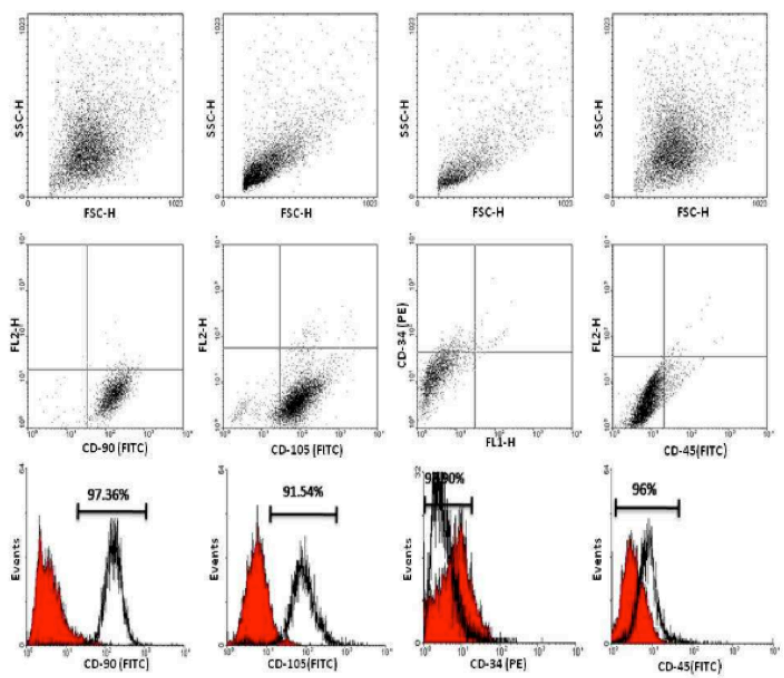

Figure 2. Immuno-phenotypic Study of the Human MSCs. MSCs derived from fourth passaged after staining with specific conjugated monoclonal antibodies with fluorochromes with specific isotype controls, were analyzed by flow cytometry. CD90 and CD105 were considered as MSCs marker, as well as CD45 and CD34 were considered as hematopoietic cell markers morphology gradually changed. In osteogenic medium, cells were transformed from spindle-shaped cells to the larger multifaceted cells, which showed a lot of sediment deposition of calcium in the cytoplasm. While in the osteoblastic medium, cells become more rounded and lost their appendages, and cavities were formed within the cell that presented as one to several holes. The mortality rate

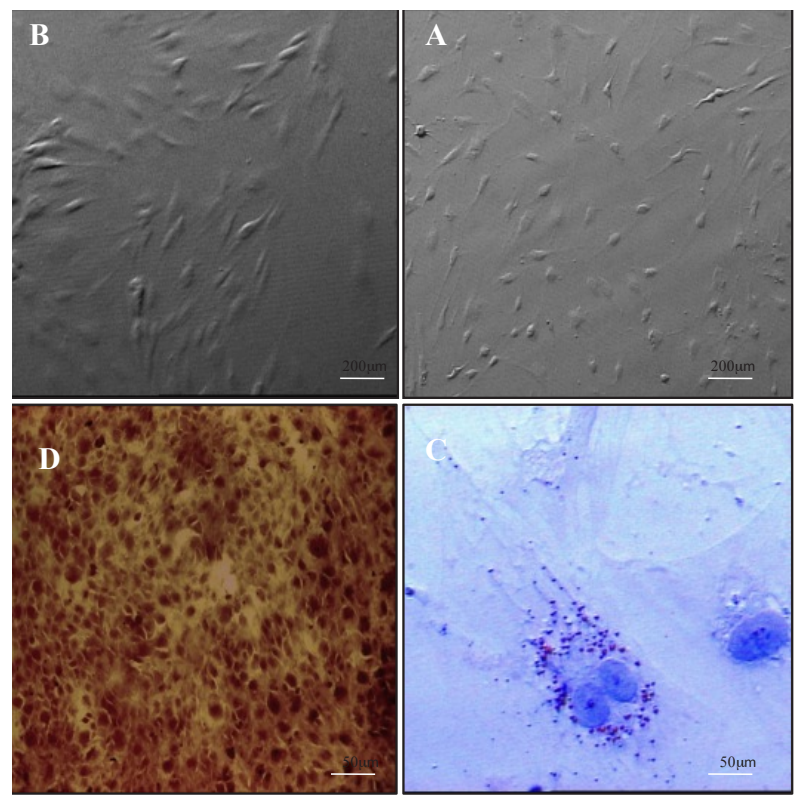

Figure 3. AD-MSCs Differentiation to the Osteogenic and Adipogenic Lineages During Fourth Passage. A, AD-MSCs after a week of adipogenic differentiation; B, ADMSCs after a week of osteogenic differentiation; C, Adipose cells derived from AD-MSCs (Oil Red-O staining); D bone cell derived from AD-MSCs (Alizarin Red- staining)
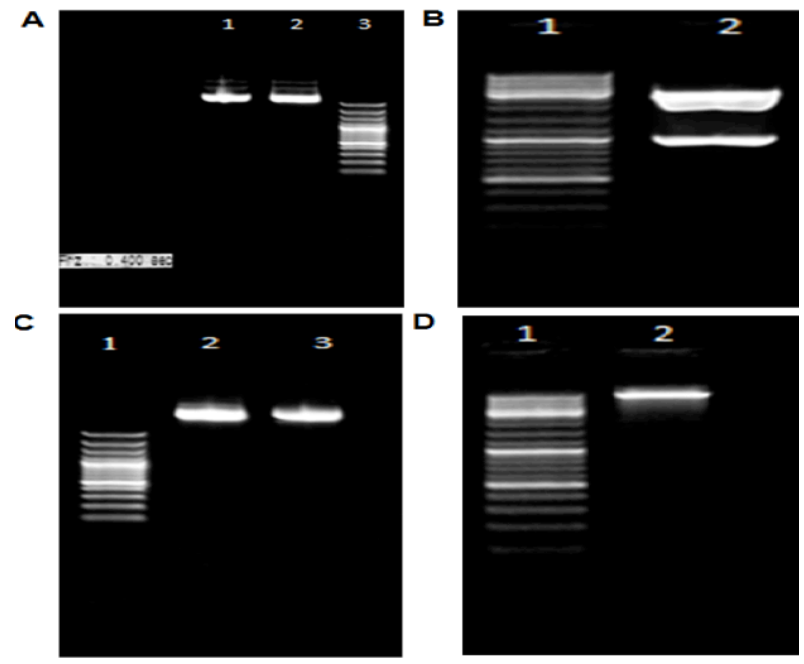

Figure 4. Plasmid Extraction and Purification. A, lanes 1 and 2: proliferation of positive bacterial colonies, lane 3: Ladder with 100bp components as standard. B, digestion of pGH plasmid with BamI and EcoRI: lane 1, ladder with 100pb components as standard, lane 2: confirmation of the pGH plasmid fragment 989bp. C, sustained expressional LeGO-IG plasmid: lane 1, Ladder with 100bp components as standard, lanes 3 and 2: plasmid purified from positive bacterial colonies. D, the second digestion of LeGo plasmid with BamI and EcoRI: lane 1, ladder with $100 \mathrm{bp}$ components as standard, lane 2 , confirmation of the presence of expressional LeGO plasmid 


\section{Armita Valizadeh et al}

in these cells was lower than the differentiated cells into bone. Also, the early differentiation was presented more in the osteoblastic medium than the osteogenic one. Vacuoles were appeared in the first 13-14 days of differentiation, while bone cells formation was taking place almost a week later (Figure 3).

\section{Extraction of $p G H$ plasmid}

After digestion, gel electrophoresis was performed on all product of a gene of interest, which showed a frond band of $989 \mathrm{pb}$ (Figure 4A); Amplification and purification of expression vectors (LeGO-IG2) was done in low-scale (Figure 4B); After purification of LeGO-IG2 plasmid, it was digested by the BamI and EcoRI enzymes, was run on agarose gel $1 \%$ (Figure 4C).

Figure 5. ?? [PLEASE SUPPLY]

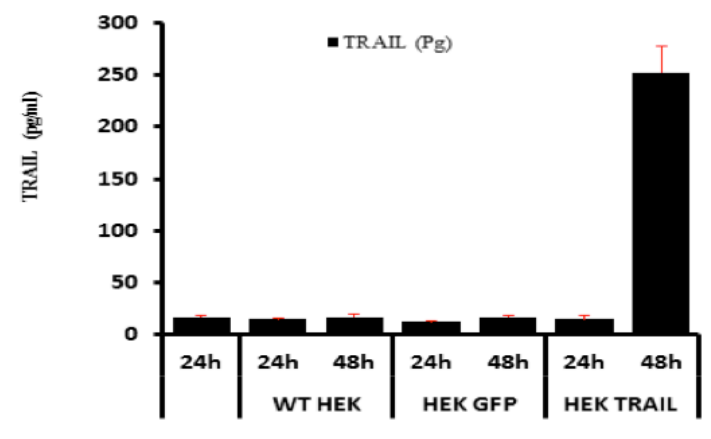

A

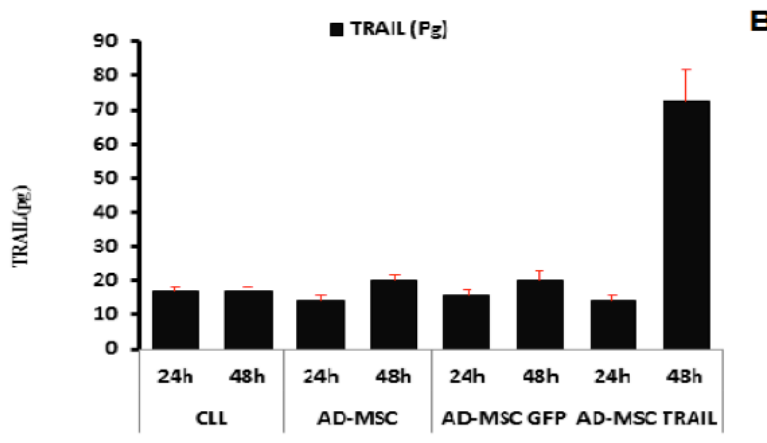

Figure 6. A, The Concentration of Secreted TRAIL Proteins in Different HEK Groups Using ELISA kit; B, The Concentration of Secreted TRAIL Proteins in AD-MSCs Group in Second (24 hours) and third (48 Hours) Days Using ELISA kit
TRAIL secretion of AD-MSCs in the HIKT293 Groups

on second day, the TRAIL in both transduct and nontransduct groups was low $(12.3 \pm 0.5 \mathrm{pg} / \mathrm{mL})$, but in the day 3, TRAIL increased significantly in the HEKTRAIL group $(253 \pm 25.1 \mathrm{pg} / \mathrm{mL})$, while no significant change was observed in in the rest of the groups (Figure 6A). The soluble TRAIL medium was very small in all groups on the second day $(14.3 \mathrm{pg} / \mathrm{mL})$, but on day 3 it was increased significantly in AD-MSC TRAIL group (72.7 pg/mL), therefore the rest of the group had no significant change. Besides there was a significant difference in the TRAIL on the third day compared to the second day in AD-MSC TRAIL group, while the difference was not significant in other groups, (Figure 6B).

GFP expression in HEKT293 groups using an inverted microscope

The observation of HEKT293 cells in groups 1 and 2 with the vector encoding the gene TRAIL containing protein GFP reporter transduct using an inverted fluorescent microscope, has revealed green spots. These spots increased in the third day, and reach to its highest amount in the period 24-48 hours. So, the strength and

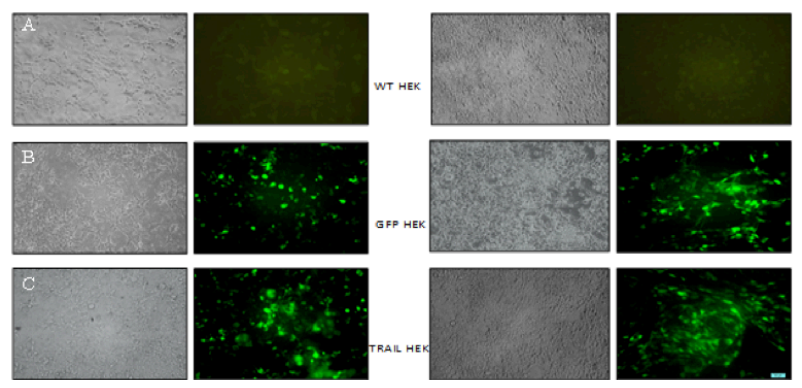

Figure 7. The Image of Protein GFP Reporter Cells in CLL and WT-HEK Cells. (A), CLL cells with HEK-GFP (B), CLL cells with HEK-TRAIL (C) a ratio of 5:1 of HEK cells to cancer cells. The presence of fluorescent cells on day 2 in the right and even more in left on day 3 (scale bar, $500 \mu \mathrm{m}$ )

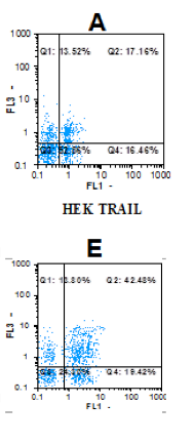

AD-MSC TRAIL
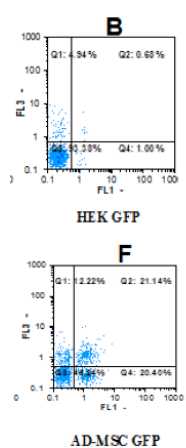

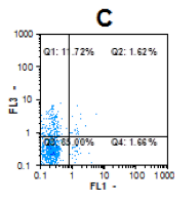

WT HEK

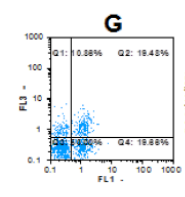

AD-MSC

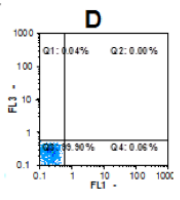

CLL

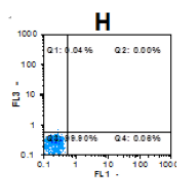

CLL
Figure 8. Results of FACS were as Follows: (Top row): The Mean Rate of HEK-TRAILApoptosis was $17.16 \%$. (A); the mean rate of apoptosis in HEK-GFP was $2.6 \%$ (B); the mean rate of WT-HEK apoptosis has been demonstrated as $2 \%$ (C); the mean rate of apoptosis in CLL group was $0.01 \%$ (D). The other results of FACS were as follows (Bottom row): the mean apoptosis in AD-MSC TRAIL group was 42\% (E); the mean apoptosis in AD-MSC GFP group was $21 \%(\mathrm{~F})$; the mean apoptosis in AD-MSC group was $19 \%(\mathrm{G})$; the mean rate of apoptosis in CLL group was $0.01 \%(\mathrm{H})$ 
TNF-Producing Mesenchymal Stem Cells and Apoptosis of Chronic B-Lymphocytic Tumor Cells Resistant to Chemotherapy
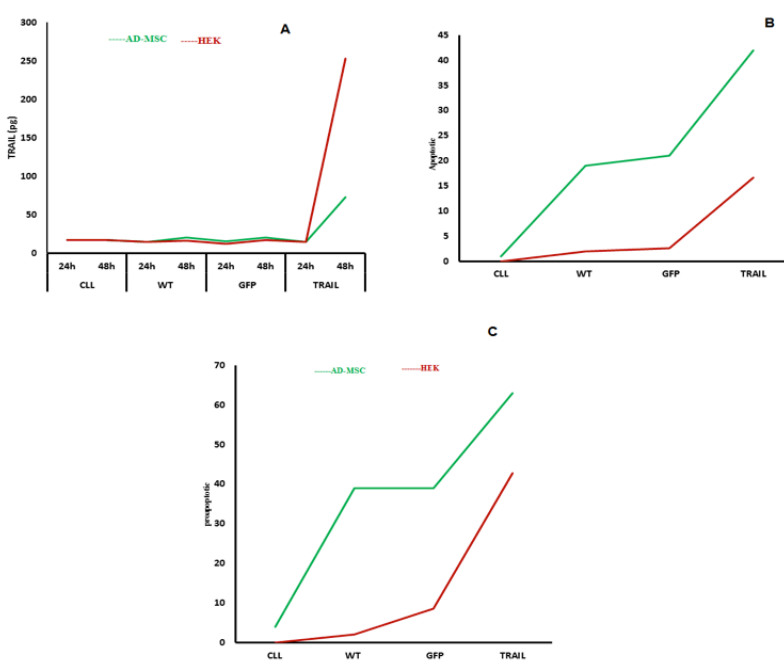

Figure 9. A Comparing the Secretion of TRAIL in Mesenchyme Feeder and HEK Feeder Groups on Day 2 (24 hours) and Day 3 (48 hours); B The Rate of Apoptosis in Various HEK and AD-MSC Groups; C Comparing the Pro-apoptotic Results Between HEK and AD-MSC Feeder Groups

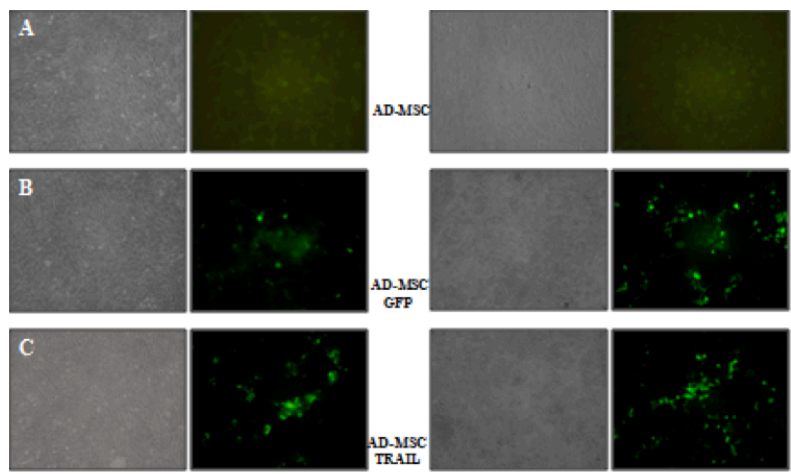

Figure 10. Image of Protein Reporter of GFP with Phase Contrast and Fluorescent Microscope CLL and AD-MSC Groups. In CLL with AD-MSC-GFP cells (A), in CLL with AD-MSC-TRAIL cells (B) in AD-MSC to CLL with the rate of 5:1 (C) The presence of fluorescent cells on day 2 (Left) and and even more expressed on day 3 (right) (Scale bar, $500 \mu \mathrm{m}$ )

adhesion of the cells to the floor plate gradually decreased, as well as virus production and the expression of GFP showed a decreasing trend (Figure 7).

\section{Apoptosis in HEKT293 groups}

There was a significant difference in the rate of proapoptosis and apoptosis in HEK-TRAIL group compared to the other groups in term of cancer cells $(\mathrm{P} \leq 0.05)$. The results prove that pro-apoptosis and apoptosis in the three groups with non-modified TRAIL gene had significant differences (Figure 8).

Comparison of TRAIL secretion in mesenchyme Feeder and HEK Feeder groups

TRAIL secretion in TRAIL-HEK within both 24 hours and 48 hours was significantly different compared to ADMSC-TRAIL (both $\mathrm{P} \leq 0.05$, Figure 9A). The information obtained from flow cytometry analysis showed that compared different groups of cells, MSC apoptosis was higher than the other groups, of which this difference in all groups were significantly higher in all MSC groups compared with the HEK groups (Figure 9B). Pro-apoptotic result in different groups of HEK and AD-MSC, shows significant differences exist $(\mathrm{P} \leq 0.05)$ in the MSC group compared to the group of HEK group. Besides, Proapoptotic result in all MSC groups was higher than HEK groups (Figure 9C).

Evaluation of GFP expression in mesenchymal groups using an inverted microscope

The results showed that in groups 1 and 2 with the TRAIL gene encoding and GFP transduct vectors, green spots can be seen due to GFP expression, which these spots present a little more on the third day, and gradually increased in the period 24-48 hours (Figure 10), but the green spots are lower compared with the HEK transduct group.

\section{Discussion}

Recent studies have shown the behavior of tumor cells is not determined only by the tumor cells, and the role of cytokines and non-tumor cells at the tumor development and progression site has attracted the attention of many researchers (Calon et al., 2012). Tumors have various recovery behaviors, and inflammation plays a major role in tumor development, progression, and metastasis (Whiteside et al., 2008; Wu et al., 2012).

Since the effect of TRAIL secreted by human mesenchymal cells in apoptosis has been reported so far, many studies have shown that cells mesenchymal or stromal-differentiated cells have an important supportive role in the hematopoiesis and prevent various diseases ( Batsali et al., 2013; Hoch et al., 2014; Sharma et al., 2014). However, abnormal mesenchymal cells may be involved in hematological diseases, especially acute myelogenous (Kojima et al., 2011) and chronic myeloid (Balakrishnan et al., 2010) leukemia. The inhibitory effects of human MSCs on tumor growth and metastasis are the research topics of interest in recent years. The aim of the present study was to assess the role of tumor necrosis factor-producing MSC on apoptosis of B-CLL cells resistant to fludarabine-based chemotherapy, and showed that a designed recombinant lentivirus structure was capable to express a secretory protein, which serve as a ligand able to bind to receptors on the membrane of cancer cells and inhibits the growth and proliferation of these cells.

Soluble TRAIL in our AD-MSC-TRAIL study group was lower than the Grisendi et al. (Grisendi et al., 2010) study that can be due to non-titration of the virus, because the infectious ability of a virus closely related to its concentration (Tiscornia et al., 2006). Titration-related increasing the concentration of the virus increases the efficiency of transduction and induces more cells to release the TRAIL. In the present study, secretory protein was the highest in HEK-TRAIL group compared to all other groups, and previous studies also have subsequent transfection efficiency in HEK cell-lines higher than the 
other cell types (Foss et al., 2000).

In the Grisendi et al. (2010) study, soluble TRAIL in GFP vector and other transducted groups were not assessable at 24 and 48 hours (Grisendi et al., 2010). However, in the present study the TRAIL was assessable in culture medium of HEK and mesenchymal groups, which may suggest that all cells release a very small amount of TRAIL.

In the present study, data showed that CLL cells are sensitive to TRAIL-mediated apoptosis, so that with more time more cellular debrides could be seen, which flow cytometry also confirmed the CLL apoptosis. Besides, in the present study cancer cells were only exposed 24 hours to the TRAIL, of which HEK-TRAIL and AD-MSC-TRAIL groups showed higher apoptosis and pro-apoptosis. These findings were different with the previous studies (Grisendi et al., 2010). However, these differences can be due to higher levels of TRAIL in cell culture and also the low impact of CLL cells to TRAIL, which is not sufficient to induce apoptosis. Also, perhaps these differences were because of the low proximity of the cancer cells with TRAIL or change of the feeder cells to cancer cells ratio, which means HEK cells are not sufficient to feed the tumor. Thus, with the increase in feeder cells to cancer cells ration may expose cancer cells to more TRAIL.

An interesting point in this study was the rate of apoptosis and pro-apoptosis in all mesenchymal feeder groups was higher than HEK feeder groups, it could be related to the plasticity of mesenchymal cells that activate different signaling pathways can inhibit tumor cells (Sen et al., 2001).

In conclusion, At the end of the study, it can be concluded that MSCs, the cells that easily and in large amounts could be separated of adipose tissue, after more than two months of post-separation are rapidly proliferating in vitro. These rapidly proliferating cells facilitate accessibility to a huge source of cells, especially when a large number of cells for cell therapy are required. These cells can carry antitumor molecules to the tumor site.

Besides, taking advantages of lentivirus, gene of interest can insert into MSCs without any change in its phenotype or cell differentiation power. In the present study, a recombinant lentivirus was constructed that can express a secretory protein, of which this protein as a ligand capable to bind to a specific receptor on the cancer cells, and inhibits the growth and proliferation of these cells.

Recombinant viral vectors adipose mesenchymal cells to provide a suitable solution to apoptosis and may have taken a small step in the treatment of this disease, as defined in our study using the plasmid encoding the TRAIL apoptosis in leukemia cell chronic lymphoid significantly be. Besides, in this study the apoptosis in cells has happened although its rate was low, using the viral delivery systems, changing the time period of exposure and accompaniment with other tumor-suppressor genes, can increase the rate of apoptosis. Finally, using recombinant viral vectors to deliver AD-MSC may consider as a suitable solution and perhaps a small step in the treatment for apoptosis of tumor cells. In this study, we used a plasmid encoding the TRAIL significantly induces apoptosis in CLL.

\section{Acknowledgements}

This paper is extracted from Ph.D. thesis of Armita Valizadeh .We thank the Ahvaz Jundishapur University of Medical Sciences (AJUMS) to financial support.

\section{References}

Abdul Halim NS, Fakiruddin KS, Ali SA, et al (2014). A comparative study of non-viral gene delivery techniques to human adipose-derived mesenchymal stem cell. Int $\mathrm{J} \mathrm{Mol}$ Sci, 15, 15044-60.

Ashkenazi A, Holland P, Eckhardt SG (2008). Ligand-based targeting of apoptosis in cancer: the potential of recombinant human apoptosis ligand 2/Tumor necrosis factor-related apoptosis-inducing ligand (rhApo2L/TRAIL). J Clin Oncol, 26, 3621-30.

Balakrishnan K, Burger JA, Quiroga MP, et al (2010). Influence of bone marrow stromal microenvironment on forodesineinduced responses in CLL primary cells. Blood, 116, 1083-91.

Batsali AK, Kastrinaki MC, Papadaki HA, et al (2013). Mesenchymal stem cells derived from Wharton's Jelly of the umbilical cord: biological properties and emerging clinical applications. Curr Stem Cell Res Ther, 8, 144-55.

Calon A, Espinet E, Palomo-Ponce S, et al (2012). Dependency of colorectal cancer on a TGF-beta-driven program in stromal cells for metastasis initiation. Cancer Cell, 22, 571-584.

de Vries EG, TimmerT, Mulder NH, et al (2003). Modulation of death receptor pathways in oncology. Drugs Today (Barc), 39, 95-109.

Foss DL, Murtaugh MP (2000). Mechanisms of vaccine adjuvanticity at mucosal surfaces. Anim Health Res Rev, 1, 3-24.

Grisendi G, Bussolari R, Cafarelli L, et al (2010). Adiposederived mesenchymal stem cells as stable source of tumor necrosis factor-related apoptosis-inducing ligand delivery for cancer therapy. Cancer Res, 70, 3718-29.

Hallek M (2015). Chronic lymphocytic leukemia: 2015 Update on diagnosis, risk stratification, and treatment. Am J Hematol, 90, 446-60.

Hallek M, Cheson BD, Catovsky D, et al (2008). Guidelines for the diagnosis and treatment of chronic lymphocytic leukemia: a report from the international workshop on chronic lymphocytic leukemia updating the national cancer institute-working group 1996 guidelines. Blood, 111, 54465456.

Hoch AI, Leach JK (2014). Concise review: optimizing expansion of bone marrow mesenchymal stem/stromal cells for clinical applications. Stem Cells Transl Med, 3, 643-652.

Kay NE, Shanafelt TD, Strege AK, et al (2007). Bone biopsy derived marrow stromal elements rescue chronic lymphocytic leukemia B-cells from spontaneous and drug induced cell death and facilitates an "angiogenic switch". Leuk Res, 31, 899-906.

Kojima K, McQueen T, Chen Y, et al (2011). p53 activation of mesenchymal stromal cells partially abrogates microenvironment-mediated resistance to FLT3 inhibition in AML through HIF-1alpha-mediated down-regulation of CXCL12. Blood, 118, 4431-9. 
TNF-Producing Mesenchymal Stem Cells and Apoptosis of Chronic B-Lymphocytic Tumor Cells Resistant to Chemotherapy

Loebinger MR, Janes SM (2010). Stem cells as vectors for antitumour therapy. Thorax, 65, 362-9.

Mahalingam D, Szegezdi E, Keane M, et al (2009). TRAIL receptor signalling and modulation: Are we on the right TRAIL? Cancer Treat Rev, 35, 280-8.

Pachon-Pena G, Yu G, Tucker A, et al (2011). Stromal stem cells from adipose tissue and bone marrow of age-matched female donors display distinct immunophenotypic profiles. J Cell Physiol, 226, 843-51.

Sadighi S, Khoshzban A, Tavakoli A, et al (2014). Isolation, amplification and identification of mesenchymal stem cells de-rived from human adipose tissue. Tehran Univ Med J, 72, 27-32.

Sen A, Lea-Currie YR, Sujkowska D, et al (2001). Adipogenic potential of human adipose derived stromal cells from multiple donors is heterogeneous. J Cell Biochem, 81, 312-9.

Sharma RR, Pollock K, Hubel A, et al (2014). Mesenchymal stem or stromal cells: a review of clinical applications and manufacturing practices. Transfusion, 54, 1418-37.

Tiscornia G, Singer O, Verma IM (2006). Production and purification of lentiviral vectors. Nat Protoc, 1, 241-5.

Walczak H, Haas TL (2008). Biochemical analysis of the native TRAIL death-inducing signaling complex. Methods $\mathrm{Mol}$ Biol, 414, 221-39.

Whiteside TL (2008). The tumor microenvironment and its role in promoting tumor growth. Oncogene, 27, 5904-12.

Wiley SR, Schooley K, Smolak PJ, et al (1995). Identification and characterization of a new member of the TNF family that induces apoptosis. Immunity, 3, 673-82.

Wu J, Li J, Salcedo R, et al (2012). The proinflammatory myeloid cell receptor TREM-1 controls Kupffer cell activation and development of hepatocellular carcinoma. Cancer Res, $\mathbf{7 2}$, 3977-86. 